\title{
Some Relationships between the Generalized Apostol- Bernoulli and Apostol-Euler Polynomials
}

\author{
Burak Kurt* \\ Department of Mathematical Education, Faculty of Educations, Akdeniz University, TR-07058 Antalya, Turkey \\ *Corresponding author: burakkurt@akdeniz.edu.tr
}

Received October 15, 2013; Revised November 25, 2013; Accepted December 03, 2013

\begin{abstract}
The main objective of this paper is to introduce and investigate two new classes of generalized ApostolBernoulli polynomials $\mathcal{B}_{n}^{[m-1, \alpha]}(x ; c, a ; \lambda)$ and Apostol-Euler polynomials $\mathcal{E}_{n}^{[m-1, \alpha]}(x ; c, a ; \lambda)$. In particular, we obtain addition formula for the new class of the generalized Apostol-Bernoulli polynomials. We also give some recurrence relations and Raabe relations for these polynomials.
\end{abstract}

Keywords: Bernoulli polynomials and numbers, Apostol-Bernoulli polynomials, Apostol-Euler polynomials, Generalized Apostol-Bernoulli polynomials

Cite This Article: Burak Kurt, "Some Relationships between the Generalized Apostol-Bernoulli and Apostol-Euler Polynomials." Turkish Journal of Analysis and Number Theory 1, no. 1 (2013): 54-58. doi: 10.12691/tjant-1-1-11.

\section{Introduction, Definitions}

Bernoulli polynomials play an important role in various expansions and approximation formulas which are useful both in analytic theory of numbers and the classical and the numerical analysis. These polynomials can be defined by various methods depending on the applications. There are six approaches to the theory of Bernoulli polynomials. We prefer here the definition by generating functions given by Euler [4].

The classical Bernoulli polynomials and the classical Euler polynomials are defined respectively as

$$
\begin{gathered}
\sum_{n=0}^{\infty} B_{n}(x) \frac{t^{n}}{n !}=\frac{t e^{x t}}{e^{t}-1},|t|<2 \pi, \\
\sum_{n=0}^{\infty} E_{n}(x) \frac{t^{n}}{n !}=\frac{2 e^{x t}}{e^{t}+1},|t|<\pi .
\end{gathered}
$$

The corresponding Bernoulli numbers $B_{n}$ and Euler numbers $E_{n}$ are given by

$$
\begin{aligned}
& B_{n}:=B_{n}(0)=(-1)^{n} B_{n}(1), \\
& E_{n}:=2^{n} E_{n}\left(\frac{1}{2}\right), n \in \mathbb{N}_{0}=\{0\} \cup\{\mathbb{N}\} .
\end{aligned}
$$

From (1.1) and (1.2), we easily derive that

$$
\begin{aligned}
& B_{n}(x+1)=\sum_{k=0}^{n}\left(\begin{array}{l}
n \\
k
\end{array}\right) B_{k}(x), \\
& E_{n}(x+1)=\sum_{k=0}^{n}\left(\begin{array}{l}
n \\
k
\end{array}\right) E_{k}(x),
\end{aligned}
$$

$$
\begin{gathered}
B_{n}(x+y)=\sum_{k=0}^{n}\left(\begin{array}{l}
n \\
k
\end{array}\right) B_{k}(x) y^{n-k}, \\
E_{n}(x+y)=\sum_{k=0}^{n}\left(\begin{array}{l}
n \\
k
\end{array}\right) E_{k}(x) y^{n-k}, \\
B_{n}(x+1)-B_{n}(x)=n x^{n-1}, n \geq 1, \\
E_{n}(x+1)+E_{n}(x)=2 x^{n}
\end{gathered}
$$

(for details, see $[11,12,13]$ ).

The generalized Apostol-Bernoulli polynomials $B_{n}^{(\alpha)}(x ; \lambda)$ order $\alpha \in \mathbb{C}$ and the generalized ApostolEuler polynomials $E_{n}^{(\alpha)}(x ; \lambda)$ order $\alpha \in \mathbb{C}$ are defined respectively by the following generating functions

$$
\begin{gathered}
\sum_{n=0}^{\infty} B_{n}^{(\alpha)}(x ; \lambda) \frac{t^{n}}{n !}=\left(\frac{t}{\lambda e^{t}-1}\right)^{\alpha} e^{x t}, \\
|t+\ln \lambda|<2 \pi, 1^{\alpha}:=1, \\
\sum_{n=0}^{\infty} E_{n}^{(\alpha)}(x ; \lambda) \frac{t^{n}}{n !}=\left(\frac{2}{\lambda e^{t}+1}\right)^{\alpha} e^{x t}, \\
|t+\ln \lambda|<\pi, 1^{\alpha}:=1 .
\end{gathered}
$$

Recently, Srivastava et. al. in $([13,14,15])$ have investigated some new classes of Apostol-Bernoulli, Apostol-Euler polynomials with parameters a, b, and c. They gave some recurrence relations and proved some theorems.

For $\lambda=1$ one can obtain the classical polynomials (1.1) and (1.2). Other generalizations can be developed as well. 
Definition 1. [Natalini [12] and S. Chen et al. [3]] The generalized Bernoulli polynomials $\mathcal{B}_{n}^{[m-1]}(x), m \geq 1$ are defined, in a suitable neigbourhood of $t=0$, by means of the generating functions

$$
G^{[m-1]}(x, t)=\frac{t^{m} e^{x t}}{e^{t}-\sum_{h=0}^{m-1} \frac{t^{h}}{h !}}=\sum_{n=0}^{\infty} \mathcal{B}_{n}^{[m-1]}(x) \frac{t^{n}}{n !} .
$$

From (1.7) for $m=1$, we obtain the generating function $G^{[0]}(x, t)=\frac{t}{e^{t}-1} e^{x t}$ of classical Bernoulli polynomials $\mathcal{B}_{n}^{[0]}(x)$. From (1.7) for $x=0$, we obtain the generalized Bernoulli numbers $\mathcal{B}_{n}^{[m-1]}$.

Definition 2. [Kurt [9]] For $m \in \mathbb{N}$, the generalized Bernoulli polynomials $\mathcal{B}_{n}^{[m-1, \alpha]}(x)$ of order $\alpha \in \mathbb{C}, m \in \mathbb{N}$ are defined by means of the generating function

$$
\begin{aligned}
G^{[m-1, \alpha]}(x, t) & =\left(\frac{t^{m}}{e^{t}-\sum_{h=0}^{m-1} \frac{t^{h}}{h !}}\right)^{\alpha} e^{x t} \\
& =\sum_{n=0}^{\infty} \mathcal{B}_{n}^{[m-1, \alpha]}(x) \frac{t^{n}}{n !}
\end{aligned}
$$

in suitable neigbourhood of $t=0$.

The case $\alpha=1$ was first introduced by Natalini and Bernardini [6]. For $\alpha=m=1$, we obtain classical Bernoulli polynomials.

By the same motivation, the generalized Euler polynomials $\mathcal{E}_{n}^{[m-1, \alpha]}(x)$ of order $\alpha \in \mathbb{C}$ and generalized Euler numbers $\mathcal{E}_{n}^{[m-1, \alpha]}$ of order $\alpha \in \mathbb{C}$ were defined by the author [10]

$$
\left(\frac{2^{m}}{e^{t}+\sum_{h=0}^{m-1} \frac{t^{h}}{h !}}\right)^{\alpha} e^{x t}=\sum_{n=0}^{\infty} \mathcal{E}_{n}^{[m-1, \alpha]}(x) \frac{t^{n}}{n !}
$$

and

$$
\left(\frac{2^{m}}{e^{t}+\sum_{h=0}^{m-1} \frac{t^{h}}{h !}}\right)^{\alpha}=\sum_{n=0}^{\infty} \mathcal{E}_{n}^{[m-1, \alpha]} \frac{t^{n}}{n !}
$$

From (1.9) and (1.10) and for $\alpha=m=1$, we obtain classical Euler polynomials and classical Euler numbers respectively:

$$
\mathcal{E}_{n}^{[0,1]}(x)=E_{n}(x), 2^{n} \mathcal{E}_{n}^{[0,1]}\left(\frac{1}{2}\right)=E_{n} .
$$

By the same motivation, the generalized Genocchi polynomials $\mathcal{G}_{n}^{[m-1, \alpha]}(x)$ of order $\alpha \in \mathbb{C}$ and generalized Genocchi numbers $\mathcal{G}_{n}^{[m-1, \alpha]}$ of order $\alpha \in \mathbb{C}$ can be defined as

$$
\left(\frac{2^{m} t^{m}}{e^{t}+\sum_{h=0}^{m-1} \frac{t^{h}}{h !}}\right)^{\alpha} e^{x t}=\sum_{n=0}^{\infty} \mathcal{G}_{n}^{[m-1, \alpha]}(x) \frac{t^{n}}{n !}
$$

and

$$
\left(\frac{2^{m} t^{m}}{e^{t}+\sum_{h=0}^{m-1} \frac{t^{h}}{h !}}\right)^{\alpha}=\sum_{n=0}^{\infty} \mathcal{G}_{n}^{[m-1, \alpha]} \frac{t^{n}}{n !}
$$

\section{New Classes of Generalized Apostol- Euler Polynomials and Apostol-Bernoulli Polynomials}

The following definitions provide a natural generalization of the Apostol-Euler polynomials $\mathfrak{E}_{n}^{[m-1, \alpha]}(x ; \lambda)$ of order $\alpha \in \mathbb{C}$ and the Apostol-Bernoulli polynomials $\mathfrak{B}_{n}^{[m-1, \alpha]}(x ; \lambda)$ of order $\alpha \in \mathbb{C}$, where $m \in \mathbb{N}$.

Definition 3. We de.ne the generalized Bernoulli polynomials $\mathfrak{B}_{n}^{[m-1, \alpha]}(x ; c, a ; \lambda)$ of order $\alpha \in \mathbb{C}$ and the generalized Euler polynomials $\mathfrak{E}_{n}^{[m-1, \alpha]}(x ; c, a ; \lambda)$ of order $\alpha \in \mathbb{C}$ respectively by

$$
\begin{aligned}
& \sum_{n=0}^{\infty} \mathfrak{B}_{n}^{[m-1, \alpha]}(x ; c, a ; \lambda) \frac{t^{n}}{n !}=\left(\frac{t^{m}}{\lambda c^{t}-\sum_{h=0}^{m-1} \frac{(t \ln a)^{h}}{h !}}\right)^{\alpha} c^{x t},(2.1 \\
& \sum_{n=0}^{\infty} \mathfrak{E}_{n}^{[m-1, \alpha]}(x ; c, a ; \lambda) \frac{t^{n}}{n !}=\left(\frac{2^{m}}{\lambda c^{t}+\sum_{h=0}^{m-1} \frac{(t \ln a)^{h}}{h !}}\right)^{\alpha} c^{x t},(2.2
\end{aligned}
$$

and

$$
\sum_{n=0}^{\infty} \mathfrak{G}_{n}^{[m-1, \alpha]}(x ; c, a ; \lambda) \frac{t^{n}}{n !}=\left(\frac{2^{m} t^{m}}{\lambda c^{t}+\sum_{h=0}^{m-1} \frac{(t \ln a)^{h}}{h !}}\right)^{\alpha} c^{x t}
$$


For $\lambda=\alpha=m=1, c=e,(2.1),(2.2)$ and (2.3) reduce to classical Bernoulli polynomial, classical Euler polynomial and classical Genocchi polynomial.

From (2.1), (2.2) and (2.3), we obtain

$\mathfrak{B}_{n}^{[m-1, \alpha]}(x ; c, a ; \lambda)=\sum_{k=0}^{n}\left(\begin{array}{l}n \\ k\end{array}\right) \mathfrak{B}_{n-k}^{[m-1, \alpha]}(0 ; c, a ; \lambda)(x \ln c)^{k}$, $\mathfrak{E}_{n}^{[m-1, \alpha]}(x ; c, a ; \lambda)=\sum_{k=0}^{n}\left(\begin{array}{l}n \\ k\end{array}\right) \mathbb{E}_{n-k}^{[m-1, \alpha]}(0 ; c, a ; \lambda)(x \ln c)^{k}$,

and

$\mathfrak{G}_{n}^{[m-1, \alpha]}(x ; c, a ; \lambda)=\sum_{k=0}^{n}\left(\begin{array}{l}n \\ k\end{array}\right) \mathfrak{G}_{n-k}^{[m-1, \alpha]}(0 ; c, a ; \lambda)(x \ln c)^{k}$.

Theorem 1. Let $c \in \mathbb{R}^{+}, \alpha, \beta \in \mathbb{C}, m \in \mathbb{N}$. Then the generalized Apostol-Bernoulli polynomials $\mathfrak{B}_{n}^{[m-1, \alpha]}(x ; c, a ; \lambda)$ and the generalized Apostol-Euler polynomials $\mathfrak{E}_{n}^{[m-1, \alpha]}(x ; c, a ; \lambda)$ satisfy the following relations

$$
\begin{aligned}
& \mathfrak{B}_{n}^{[m-1, \alpha+\beta]}(x+y ; c, a ; \lambda) \\
= & \sum_{k=0}^{n}\left(\begin{array}{l}
n \\
k
\end{array}\right) \mathfrak{B}_{n-k}^{[m-1, \alpha]}(x ; c, a ; \lambda) \mathfrak{B}_{n-k}^{[m-1, \beta]}(y ; c, a ; \lambda) \\
& \mathfrak{E}_{n}^{[m-1, \alpha+\beta]}(x+y ; c, a ; \lambda) \\
= & \sum_{k=0}^{n}\left(\begin{array}{l}
n \\
k
\end{array}\right) \mathfrak{E}_{n-k}^{[m-1, \alpha]}(x ; c, a ; \lambda) \mathfrak{E}_{n-k}^{[m-1, \beta]}(y ; c, a ; \lambda)
\end{aligned}
$$

and

$$
\begin{aligned}
& \mathcal{G}_{n}^{[m-1, \alpha+\beta]}(x+y ; c, a ; \lambda) \\
= & \sum_{k=0}^{n}\left(\begin{array}{l}
n \\
k
\end{array}\right) \mathcal{G}_{n-k}^{[m-1, \alpha]}(x ; c, a ; \lambda) \mathcal{G}_{n-k}^{[m-1, \beta]}(y ; c, a ; \lambda)
\end{aligned}
$$

respectively.

Proof. Considering the generating function (2.1) and comparing the coefficients of $\frac{t^{n}}{n !}$ in the both sides of the above equation, we arrive at (2.4). Proof of (2.5) and (2.6) are similar.

Theorem 2. The generalized Apostol-Bernoulli polynomials $\mathfrak{B}_{n}^{[m-1, \alpha]}(x ; c, a ; \lambda)$ satisfy the following recurrence relation:

$$
\begin{aligned}
& \lambda \mathfrak{B}_{n}^{[m-1, \alpha]}(x+1 ; c, a ; \lambda)-\mathfrak{B}_{n}^{[m-1, \alpha]}(x ; c, a ; \lambda) \\
= & n \sum_{k=0}^{n-1}\left(\begin{array}{c}
n-1 \\
k
\end{array}\right) \mathfrak{B}_{k}^{[m-1, \alpha]}(x ; c, a ; \lambda) \mathfrak{B}_{n-1-k}^{[0,-1]}(0 ; c, a ; \lambda) .
\end{aligned}
$$

Proof. Considering the expression
$\lambda \mathfrak{B}_{n}^{[m-1, \alpha]}(x+1 ; c, a ; \lambda)-\mathfrak{B}_{n}^{[m-1, \alpha]}(x ; c, a ; \lambda)$ and using generating function (2.1), the proof follows.

Corollary 1. The generalized Apostol-Euler polynomials $\mathfrak{E}_{n}^{[m-1, \alpha]}(x ; c, a ; \lambda)$ satisfy the following recurrence relation:

$$
\begin{aligned}
& \lambda \mathfrak{E}_{n}^{[m-1, \alpha]}(x+1 ; c, a ; \lambda)+\mathfrak{E}_{n}^{[m-1, \alpha]}(x ; c, a ; \lambda) \\
= & \sum_{k=0}^{n}\left(\begin{array}{l}
n \\
k
\end{array}\right) \mathfrak{E}_{k}^{[m-1, \alpha]}(x ; c, a ; \lambda) \mathfrak{E}_{n-k}^{[0,-1]}(0 ; c, a ; \lambda) .
\end{aligned}
$$

Theorem 3. There is the following relation between the generalized Apostol-Bernoulli polynomials $\mathfrak{B}_{n}^{[0, \alpha]}(x ; c, a ; \lambda)$ for $m=1$ and the generalized ApostolEuler polynomials $\mathfrak{E}_{k}^{[0, \alpha]}(x ; c, a ; \lambda)$ for $m=1$ :

$$
\begin{aligned}
& \mathfrak{B}_{n}^{[0, \alpha]}\left(\frac{x+y}{2} ; c, a ; \lambda^{2}\right) \\
= & 2^{-n} \sum_{k=0}^{n}\left(\begin{array}{l}
n \\
k
\end{array}\right) \mathfrak{E}_{k}^{[0, \alpha]}\left(\frac{x}{2} ; c, a ; \lambda\right) \mathfrak{B}_{n-k}^{[0, \alpha]}\left(\frac{y}{2} ; c, a ; \lambda\right)
\end{aligned}
$$

Proof. We take $m=1 ; \frac{x+y}{2}, \lambda^{2}$ and $2 t$ instead of $x, \lambda$ and $t$ respectively. We write as:

$$
\begin{aligned}
& \sum_{n=0}^{\infty} \mathfrak{B}_{n}^{[0, \alpha]}\left(\frac{x+y}{2} ; c, a ; \lambda^{2}\right) \frac{2^{n} t^{n}}{n !} \\
= & \left(\frac{2^{m}}{\lambda c^{t}+1}\right)^{\alpha} c^{\frac{x}{2} t}\left(\frac{t^{m}}{\lambda c^{t}-1}\right)^{\alpha} c^{\frac{y}{2} t} \\
= & \left(\sum_{n=0}^{\infty} \mathfrak{E}_{n}^{[0, \alpha]}\left(\frac{x}{2} ; c ; \lambda\right) \frac{t^{n}}{n !}\right)\left(\sum_{l=0}^{\infty} \mathfrak{B}_{l}^{[0, \alpha]}\left(\frac{y}{2} ; c ; \lambda\right) \frac{t^{l}}{l !}\right) \\
= & \sum_{n=0}^{\infty}\left(2^{-n} \sum_{k=0}^{n}\left(\begin{array}{l}
n \\
k
\end{array}\right) \mathfrak{E}_{k}^{[0, \alpha]}\left(\frac{x}{2} ; c ; \lambda\right) \mathfrak{B}_{n-k}^{[0, \alpha]}\left(\frac{y}{2} ; c ; \lambda\right)\right) \frac{t^{n}}{n !} .
\end{aligned}
$$

Comparing the coefficients of $\frac{t^{n}}{n !}$, we obtain (2.9).

Theorem 4. The generalized Apostol-Bernoulli polynomials $\mathfrak{B}_{n}^{[m-1, \alpha]}(x ; c, a ; \lambda)$ satisfy the following recurrence relation:

$$
\begin{aligned}
& \mathfrak{B}_{n}^{[0, \alpha-\beta]}(x ; c, a ; \lambda) \\
= & (-1)^{p} \frac{n !}{(n+p) !}\left\{\mathfrak{B}_{n+p}^{[0, \alpha]}(x ; c, a ; \lambda)+\sum_{k=0}^{n+p}\left(\begin{array}{c}
n+p \\
k
\end{array}\right) \sum_{r=0}^{p}\left(\begin{array}{l}
p \\
r
\end{array}\right)(2.1\right. \\
& \left.\times(-1)^{r} \lambda^{r} \mathfrak{B}_{k}^{[0, \alpha]}(x ; c, a ; \lambda)(r \ln c)^{n+p-k}\right\} .
\end{aligned}
$$

Proof. From (2.1) for $m=1$, we write as

$$
\begin{gathered}
\sum_{n=0}^{\infty} \mathfrak{B}_{n}^{[0, \alpha-p]}(x ; c, a ; \lambda) \frac{t^{n}}{n !}=\left(\frac{t}{\lambda c^{t}-1}\right)^{\alpha-p} c^{x t} \\
=\left(\frac{\lambda c^{t}-1}{t}\right)^{p}\left(\sum_{n=0}^{\infty} \mathfrak{B}_{n}^{[0, \alpha]}(x ; c, a ; \lambda) \frac{t^{n}}{n !}\right) \\
t^{p} \sum_{n=0}^{\infty} \mathfrak{B}_{n}^{[0, \alpha-p]}(x ; c, a ; \lambda) \frac{t^{n}}{n !} \\
=\left(\lambda c^{t}-1\right)^{p}\left(\sum_{k=0}^{\infty} \mathfrak{B}_{k}^{[0, \alpha]}(x ; c, a ; \lambda) \frac{t^{k}}{k !}\right) \\
\left(1-\lambda e^{t \ln c}\right)^{p}=\sum_{r=0}^{p}\left(\begin{array}{l}
p \\
r
\end{array}\right)(-1)^{r} \lambda^{r} \sum_{l=0}^{\infty}(r \ln c)^{l} \frac{t^{l}}{l !} \\
=1+\sum_{l=0}^{\infty} \sum_{r=0}^{p}\left(\begin{array}{l}
p \\
r
\end{array}\right)(-1)^{r} \lambda^{r}(r \ln c)^{l} \frac{t^{l}}{l !} .
\end{gathered}
$$


We put (2.12) in the right hand side of (2.11). Then

$$
\begin{aligned}
& (-1)^{p} \sum_{n=p}^{\infty} \mathfrak{B}_{n-p}^{[0, \alpha-p]}(x ; c, a ; \lambda) \frac{t^{n}}{(n-p) !} \\
= & \sum_{n=0}^{\infty}\left(\begin{array}{c}
\mathfrak{B}_{n}^{[0, \alpha]}(x ; c, a ; \lambda) \\
\left.+\sum_{k=0}^{\infty}\left(\begin{array}{l}
n \\
k
\end{array}\right) \sum_{r=0}^{p}\left(\begin{array}{l}
p \\
r
\end{array}\right)(-1)^{r} \lambda^{r} \mathfrak{B}_{k}^{[0, \alpha]}(x ; c, a ; \lambda)(r \ln c)^{n-k}\right) \frac{t^{n}}{n !}
\end{array}\right)
\end{aligned}
$$

If we make necessary operations in the last equation and comparing the coefficients of $\frac{t^{n}}{n !}$, we arrive (2.10).

Theorem 5. The following relations hold true:

$$
\begin{aligned}
& \mathfrak{E}_{n}^{[0,-\alpha]}(x ; c, a ; \lambda) \\
= & \sum_{k=0}^{\infty}\left(\begin{array}{l}
n \\
k
\end{array}\right) \mathfrak{B}_{k}^{[0,-\alpha]}\left(-w ; c, a ; \lambda^{2}\right) 2^{k} \mathfrak{B}_{n-k}^{[0, \alpha]}(x+2 w ; c, a ; \lambda)
\end{aligned}
$$

and

$$
\begin{aligned}
& \mathfrak{B}_{k}^{[0,-\alpha]}\left(x ; c, a ; \lambda^{2}\right) \\
= & 2^{-n} \sum_{k=0}^{\infty}\left(\begin{array}{l}
n \\
k
\end{array}\right) \mathfrak{B}_{k}^{[0, \alpha]}(x ; c, a ; \lambda) \mathfrak{E}_{n-k}^{[0, \alpha]}(x ; c, a ; \lambda) .
\end{aligned}
$$

Proof. From (2.1) for $m=1$,

$$
\begin{aligned}
& \sum_{n=0}^{\infty} \mathfrak{E}_{n}^{[0,-\alpha]}(x ; c, a ; \lambda) \frac{t^{n}}{n !} \\
&=\left(\frac{2 t}{\lambda^{2} c^{2 t}-1}\right)^{-\alpha} c^{2 t(-w)}\left(\frac{t}{\lambda c^{t}-1}\right)^{\alpha} c^{t(x+2 w)} \\
&=\left(\sum_{k=0}^{\infty} \mathfrak{B}_{k}^{[0,-\alpha]}\left(-w ; c, a ; \lambda^{2}\right) \frac{2^{k} t^{k}}{k !}\right) \\
& \times\left(\sum_{l=0}^{\infty} \mathfrak{B}_{l}^{[0, \alpha]}(x+2 w ; c, a ; \lambda) \frac{t^{l}}{l !}\right) \\
&= \sum_{n=0}^{\infty}\left(\sum_{k=0}^{\infty}\left(\begin{array}{l}
n \\
k
\end{array}\right) \mathfrak{B}_{k}^{[0,-\alpha]}\left(-w ; c, a ; \lambda^{2}\right)\right) \underline{t}_{n-k}^{n} \\
& {[0, \alpha] }
\end{aligned}
$$

Comparing the coefficients of $\frac{t^{n}}{n !}$, we obtain (2.13).

For the proof of (2.14), we write

$$
\begin{aligned}
& \sum_{k=0}^{\infty} \mathfrak{B}_{k}^{[0, \alpha]}\left(x ; c, a ; \lambda^{2}\right) \frac{2^{n} t^{n}}{n !} \\
= & \left(\frac{2 t}{\lambda^{2} c^{2 t}-1}\right)^{\alpha} c^{2 x t} \\
= & \left(\frac{t}{\lambda c^{t}-1}\right)^{\alpha} c^{2 x t}\left(\frac{2}{\lambda c^{t}+1}\right) c^{x t} \\
= & \sum_{k=0}^{\infty}\left(2^{-n} \sum_{k=0}^{\infty}\left(\begin{array}{l}
n \\
k
\end{array}\right) \mathfrak{B}_{k}^{[0, \alpha]}(x ; c, a ; \lambda) \mathfrak{E}_{n-k}^{[0, \alpha]}(x ; c, a ; \lambda)\right) \frac{t^{n}}{n !} .
\end{aligned}
$$

Comparing the coefficients of $\frac{t^{n}}{n !}$, we arrived to result.
Corollary 2. The new generalized Bernoulli polynomials $\mathfrak{B}_{k}^{[m-1, \alpha]}(x ; c, a)$ for $\alpha=m=1$ and the new generalized Euler polynomials $\mathfrak{E}_{k}^{[m-1, \alpha]}(x ; c, a)$ for $\alpha=m=1$ satisfy the following Raabe relations:

$$
\sum_{k=0}^{l-1} \mathfrak{B}_{n}^{[0,1]}\left(\frac{x+k}{l} ; c, a\right)=l^{1-n} \mathfrak{B}_{n}^{[0,1]}(x ; c, a)
$$

and

$$
\sum_{k=0}^{l-1} \mathfrak{E}_{n}^{[0,1]}\left(\frac{x+k}{l} ; c, a\right)=l^{-n_{\mathfrak{E}}}[0,1](x ; c, a), \text { for l odd } \text {. }
$$

Proof. We put $m=\lambda=\alpha=1$ in (2.1),

$$
\begin{aligned}
& \sum_{n=0}^{\infty} \sum_{k=0}^{l-1} \mathfrak{B}_{n}^{[0,1]}\left(\frac{x+k}{m l} ; c, a\right) \frac{t^{n}}{n !} \\
= & \sum_{k=0}^{l-1} \frac{t}{c^{t}-1} c^{t\left(\frac{x+k}{l}\right)} \\
= & \frac{t}{c^{t}-1} c^{\frac{t x}{l}} \sum_{k=0}^{l-1} e^{\left(\frac{t}{l} \ln c\right)^{k}} \\
= & l^{1-n} \sum_{n=0}^{\infty} \mathfrak{B}_{n}^{[0,1]}(x ; c, a) \frac{t^{n}}{n !} .
\end{aligned}
$$

From the last equality, we have (2.15).

Second equation of this corollary can be obtained similarly, so we omit it.

\section{Acknowledgements}

This paper was supported by the Scientific Research Project Administration of Akdeniz University.

\section{References}

[1] A. Bagdasaryan and S. Araci, Some new identities on the ApostolBernoulli polynomials higher order derived from Bernoulli basis, arXiv:1311.4148 [math.NT].

[2] G. Bretti and P. E. Ricci, Multidimensional extensions of the Bernoulli and Appell polynomials, Taiwanese J. of Math. 8, 415428, 2004.

[3] S. Chen, Yi Chai and Q.-M. Luo, An extension of generalized Apostol-Euler polynomials, Advances in Difference Equation.

[4] F. Costabile, F. Dellaccio and M. I. Gualtieri, A new approach to Bernoulli polynomials, Rendi. di. Math. Series VII, 26, 1-12, 2006.

[5] S. Gaboury and B. Kurt, Some relations involving Hermite-based Apostol-Genocchi polynomials, App. Math. Sci., 82, 4091-4102, 2012.

[6] Y. He and C. Wang, Some formulae of products of the ApostolBernoulli and Apostol-Euler polynomials, Discrete Dynamics in Nature and Society, Article ID 927953, 11 pages, 2012.

[7] T. Kim, Some identities for the Bernoulli, Euler and Genocchi numbers and polynomials, Adv. Stud. Contemp. Math. 20, 18-23, 2010 .

[8] T. Kim, T. Mansour, S.-H. Rim and S.-H. Lee, Apostol-Euler polynomials arising from umbral calculus, Advances in Difference Equations 2013, 2013:300.

[9] B. Kurt, A further generalization of the Bernoulli polynomials and on the 2D-Bernoulli polynomials $\mathrm{B}_{\mathrm{n}}{ }^{2}(\mathrm{x}, \mathrm{y})$, App. Math. Sci., 47, 2315-2322, 2010. 
[10] B. Kurt, A further generalization of the Euler polynomials and on the 2D-Euler polynomials, Proc. Jang. Math. Soc., 15, 389-394, 2012.

[11] Q.-M. Luo, The multiplication formulas for the Apostol-Bernoulli and Apostol-Euler polynomials of higher order, Int. Trans. Spec. Func. Vol 20(5), 377-391, 2009.

[12] P. Natalini and A. Bernardini, A Generalization of the Bernoulli polynomials, J. of App. Math., 153-163, 2003.

[13] H. M. Srivastava and A. Pinter, Remarks on some relationships between the Bernoulli and Euler polynomials, App. Math. Letter, $17,375-380,2004$.
[14] H. M. Srivastava, M. Garg and S. Choudhary, A new generalization of the Bernoulli and related polynomials, Russian J. of Math. Phys., 17, 251-261, 2010.

[15] H. M. Srivastava, M. Garg and S. Choudhary, Some new families of neralized Euler and Genocchi polynomials, Taiwanese J. of Math., 15, 283-305, 2011.

[16] R. Trembly, S. Gaboury and B.-J. Fugére, A new class of generalized Apostol-Bernoulli polynomials and some analogues of the Srivastava-Pinter addition theorem, Applied Math. Letter, 24, 1888-1893, 2011.

[17] R. Trembly, S. Gaboury and B.-J. Fugére, Some new classes of generalized Apostol-Euler and Apostol-Genocchi polynomials, Inter. J. of Math. and Math. Sci., 2012. 